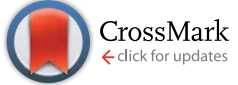

Cite this: RSC Adv., 2015, 5, 61249

Received 4th May 2015

Accepted 1st July 2015

DOI: $10.1039 / c 5 r a 08231 e$

www.rsc.org/advances

\section{Packing directed beneficial role of 3-D rigid alicyclic arms on the templated molecular aggregation problem $\uparrow$}

\begin{abstract}
Sunil Kumar, ${ }^{a}$ Punita Singh, ${ }^{\mathrm{b}}$ Ritu Srivastava ${ }^{\mathrm{b}}$ and Subrata Ghosh ${ }^{\star a}$
Subtle changes in molecular structure have been used to alter the molecular packing and optical properties of organic luminophores. Thus it is important to study simple and advantageous structural modification to overcome limitations of aggregation quenching of fluorescence. Planar conjugated organic compounds are unlikely to be used in OLED devices as they suffer with luminescence weakening due to $\pi-\pi$ cofacial stacking and excimer formation in the solid state. To avoid such critical issues, doped device architecture for OLED devices has widely been adopted which actually complicates the device fabrication process. Thus an approach for delimiting these drawbacks using simple methodologies is highly desirable for cost effective OLED device fabrication. In this context, two 3-dimensional rigid arms have been introduced into a planar benzo[h]chromen-2-one core, which suffers from aggregation caused quenching, as peripheral substituents to tune the molecular packing and subsequently their optical properties. The resultant compounds 1 and 2 have been tested for non-doped and doped OLED device application. Compound 1 was found to be highly thermally stable with a decomposition temperature of $344{ }^{\circ} \mathrm{C}$. Single crystal XRD studies helped to elucidate that voluminous ring substituents are capable of eradicating co-facial $\pi-\pi$ stacking by increasing the intermolecular distance and hence the luminescence in the solid state could be regenerated. This tuning of intermolecular distance between molecules helped to mitigate the close packed arrangement at a molecular level and also provided the bulk with the enhanced emission property. Electroluminescence from a pristine layer of compound 1 was possible. Thus an approach enabling planar luminophores to be used in OLED applications utilizing thin films of structurally engineered luminophores has been presented.
\end{abstract}

\section{Introduction}

Conjugated planar organic molecules suffer from a notorious problem of forming aggregates or $\pi-\pi$ stacks in the solid state. These molecular aggregates make luminophores less emissive in the solid state due to aggregation caused quenching (ACQ) of fluorescence. ${ }^{1}$ The ACQ is considered to be the sole reason limiting the application area of many luminophores. ${ }^{2-4}$ Thus, it is necessary to resolve this detrimental effect of aggregation in solids as solid films of luminophores for practical application in various fields which include organic light emitting diode (OLED) devices, ${ }^{5-7}$ solar energy conversion, ${ }^{8-10}$ optical data storage, ${ }^{11-13}$ organic field effect transistors (FETs), ${ }^{14-16}$ two photon absorption (TPF) $)^{11,17,18}$ and so on. It is well documented in the literature that the planar molecules become non emissive

${ }^{a}$ School of Basic Sciences, Indian Institute of Technology Mandi, Mandi-175001, H.P, India.E-mail: subrata@iitmandi.ac.in; Tel: +91-1905-267065

${ }^{b}$ Physics of Energy Harvesting Division, National Physical Laboratory, New Delhi, India $\dagger$ Electronic supplementary information (ESI) available. CCDC 1052203. For ESI and crystallographic data in CIF or other electronic format see DOI: 10.1039/c5ra08231e in the solid state due to closely spaced arrangement and $\pi-\pi$ interaction. ${ }^{19}$ Various successful procedures have been developed in the last few years to frustrate the ACQ process. These include (a) distorted/twisted luminophores; ${ }^{20-22}$ (b) long aliphatic chain substitution of the core luminophore; ${ }^{23-25}$ (c) spiro compounds $;^{26-28}$ (d) insertion of bulky substituents to the core luminophore ${ }^{29-31}$ and (e) a dipole stacking model. ${ }^{32}$ All these approaches make the luminophore highly emissive in the solid state because of prevention of aggregation quenching at a molecular level. Moreover, interesting bulk phenomena known as AIE $^{33,34}$ (aggregation induced emission) and AIEE ${ }^{35,36}$ (aggregation induced enhanced emission) have received remarkable attention from researchers. ${ }^{37-42}$ It is well established that AIE/ AIEE are bulk phenomena where molecules are capable of emitting from the aggregate state. The synthesis of AIEE active luminophores is chemically tedious which in turn adds up to additional cost for the whole process for an OLED device. On many occasions the effect of structure engineering on the optical band gap and emission wavelength of parent ACQ suffering luminophores has also been observed. Since quenching of fluorescence in the solid state is due to the reason that fluorophore molecules lie in close vicinity of each other 
providing non-radiative pathways for exciton relaxation through intermolecular interaction, it is clear that bulky substituents prevent these intermolecular interactions by providing non interacting space between molecules. Thus, a material-friendly and simple design strategy capable of reducing the intermolecular interaction problem of luminophores without affecting the band gap is highly desirable.

We recently addressed the ACQ problem via a substitution approach on a coumarin derivative and concluded that simple alkyl chains were effective in preventing the close packed arrangement of luminophores ${ }^{43}$ to some extent. Since the key idea is to increase the dimensionality of planar molecules through structure engineering, one of the possible ways to achieve this is simply to incorporate three dimensional (3-D) alicyclic ring substituents into the molecular scaffold. ${ }^{44-49}$ The effects of three dimensional alicyclic rigid systems on the solid state emission of luminescent polymers ${ }^{50}$ as pendant units have been investigated. However, to the best of our knowledge, no efforts have been directed yet to investigate the effect of rigid 3D arms on the ACQ problem/solid state emission of planar molecular systems. Coumarins have been an area of interest for many researchers over the past few decades and their potential utilities have been reviewed recently by Gryko and co-workers. ${ }^{51}$ It is well reported that coumarins lack thin film applications because of quenching of emission in the solid state mainly due to their strong intermolecular interaction and aggregation tendency ${ }^{43,52}$ Structural modifications to coumarin rings have been explored by other research groups to improve solid state emission properties. ${ }^{53,54}$ Thus a systematic study affirming the effect on solid state emission properties of planar coumarins containing different substituents which are capable of delimiting intermolecular interactions is of importance. To complete the present study on non-emissive planar benzo[h]chromene derivatives, compound $\mathbf{3}$ was chosen as key building block, and two new coumarin derivatives bearing adamantyl (compound 1) and norbornane (compound 2) rings were synthesized (Fig. 1). Herein, we report our recent achievements through detailed experimental and theoretical studies on delimiting the $\pi-\pi$ intermolecular interactions issue and restoring solid state emission of coumarins using special 3-D chemical architecture. General photophysical properties such as UV-vis absorption, photoluminescence studies, electrochemical, thermal stability and single crystal studies were investigated to support our results. It was found that these alicyclic substituents helped to mitigate the intermolecular interactions of luminophores and also provided the bulk powder with AIEE property. To fully exploit the potential utility of the present approach, OLED devices having non-doped and doped emitting layers were fabricated and studied. We would like to report that 3-D ring substitution does not alter the band gap but an effect on molecular packing and intermolecular spacing was observed which we assume is efficient in delimiting the intermolecular interaction to a greater extent. However, a little contribution of excimer and exciplex emission was observed in PL and EL data respectively, but we believe that the present approach describes a way to control the intermolecular interactions and excimer emission by utilizing bulkier 3-D aliphatic arms as substituents.
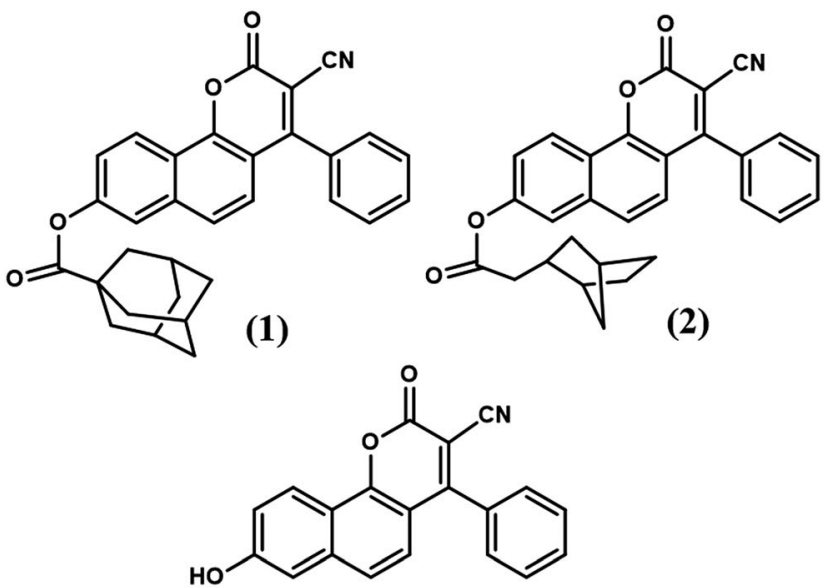

(3)

Fig. 1 Chemical structures of 1 and 2 and 3 .

\section{Results and discussion}

\subsection{Synthesis}

The desired compounds 1 and $\mathbf{2}$ (Fig. 1) were synthesized from precursor hydroxy-coumarin (3) which was reacted with 2-adamantylcarbonylchloride in dichloromethane (DCM) and norbornane-2-acetyl chloride in tetrahydrofuran (THF) solvent respectively (Scheme S1 $\dagger$ ). The structural identities of $\mathbf{1}$ and $\mathbf{2}$ have been investigated using ${ }^{1} \mathrm{H} /{ }^{13} \mathrm{C}-\mathrm{NMR}$, mass spectroscopy and FT-IR spectroscopy. The structural integrity of compound 2 was authenticated by making use of the single crystal X-ray crystallography technique.

\subsection{Optical properties}

Normalized UV-vis and PL spectra of these coumarin derivatives diluted in dichloromethane are shown in Fig. 2 (Fig. S1†). Since compounds 1 and 2 have the same coumarin core and differ only in chain substitution, both of the compounds have almost the same vibronic fine absorption continuum from $270-450 \mathrm{~nm}$ (Fig. S2 $\dagger$ ). Compound 1 and 2 showed similar PL emission centered at $462 \mathrm{~nm}$ with full width at half maximum (fwhm) of $68 \mathrm{~nm}$ and $80 \mathrm{~nm}$ for dichloromethane solution respectively. It is worth noting that PL spectra for spin coated films (Fig. 3a and b) of compounds 1 and 2 were broad and red shifted by $22 \mathrm{~nm}$ from solution PL emission (484 nm). These types of red shifts are expected for compounds for their solution and film PL emission and might be due to increased molecular interactions in the solid state, ${ }^{55}$ exciton hopping and dielectric change for solid state emission. ${ }^{56}$ Solid state quantum yield data also supported the fact that sterically bulky ring substitutions change the emission properties of these compounds. Compounds 1 and 2 were able to achieve quantum yields of 0.14 and 0.15 , while precursor coumarin 3 had a quantum yield of 0.02 only.

To comment on the fact that the PL emission depends on the solvent polarity, ${ }^{57}$ solvatochromic studies were carried out for both compounds in different solvents. Both $\mathbf{1} / \mathbf{2}$ showed similar 

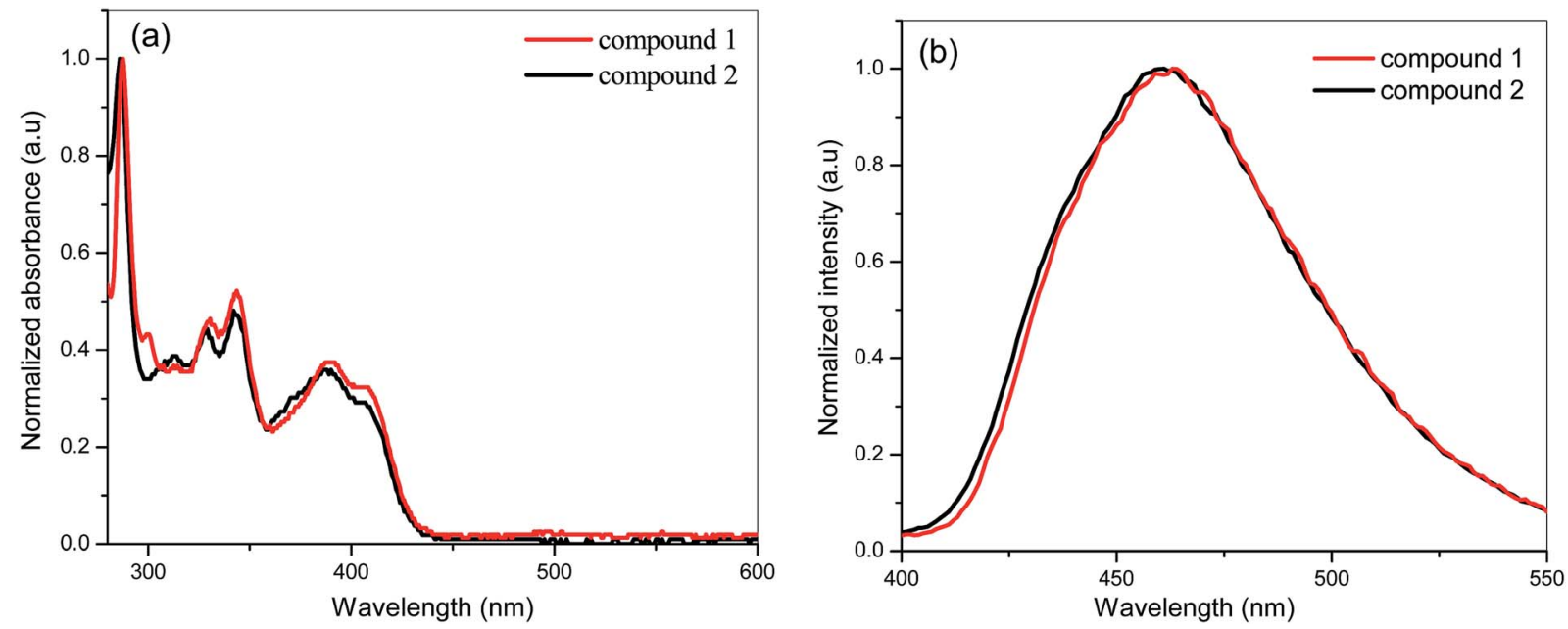

Fig. 2 (a) UV-vis and (b) PL spectra for compounds 1 and 2 in DCM.

positive solvatochromism and a red shift of $12 \mathrm{~nm}$ which was expected as we switched the solvent system from dichloromethane to dimethyl sulfoxide (DMSO) (Fig. S1†). These results suggested that the red shift in fluorescence emission is more likely due to the absorption occurring to a locally excited state which then gets interconverted into a charge-transfer state prior to emission. UV-vis solvatochromism (Fig. S2 $\dagger$ ) for both coumarin derivatives $\mathbf{1} / \mathbf{2}$ showed no dependence on solvent polarity which also explained that the dipole moment in the ground state was not affected by the polar solvents. To investigate the AIEE property of $\mathbf{1 / 2}$, PL behavior in a THF/water mixture with increasing content of water was studied. Both the compounds showed an increase in fluorescence intensity in the presence of a varying percentage of water ( $0 \%$ to $60 \%)$ in THF. Thus we could explain that the fluorescence enhancement is occurring due to the AIEE property of the studied coumarin derivatives which is plausibly due to the formation of emissive molecular aggregates. ${ }^{38-40}$ It also suggested that alicyclic 3-D substituents were helpful to get rid of the intermolecular interaction between luminophores thereby providing compounds $\mathbf{1} / \mathbf{2}$ with AIEE property. But as the content of water was increased from 80 to $90 \%$, PL intensities were found to decline. This decrease in fluorescence intensity could be attributed to the emission occurring only from the surface molecules of bigger molecular aggregates formed at higher water concentration.

\subsection{Thermal properties}

The highly stiff aromatic coumarin core structure coupled with rigid aliphatic three dimensional ring structures was expected to have good thermal stability which is quite useful for the long life of OLED device applications. ${ }^{58}$ Both the coumarin derivatives showed good thermal stabilities. Compound $\mathbf{1}$ was found to have a melting point (m.p.) at $297{ }^{\circ} \mathrm{C}$ (glass transition temperature, $T_{\mathrm{g}}=278^{\circ} \mathrm{C}$ ) with a decomposition temperature $T_{\mathrm{d}}$ (5\% weight loss) at $344{ }^{\circ} \mathrm{C}$ (Fig. 4). Unlike compound 1, compound 2 showed a lower melting point at $210{ }^{\circ} \mathrm{C}\left(T_{\mathrm{g}}=\right.$
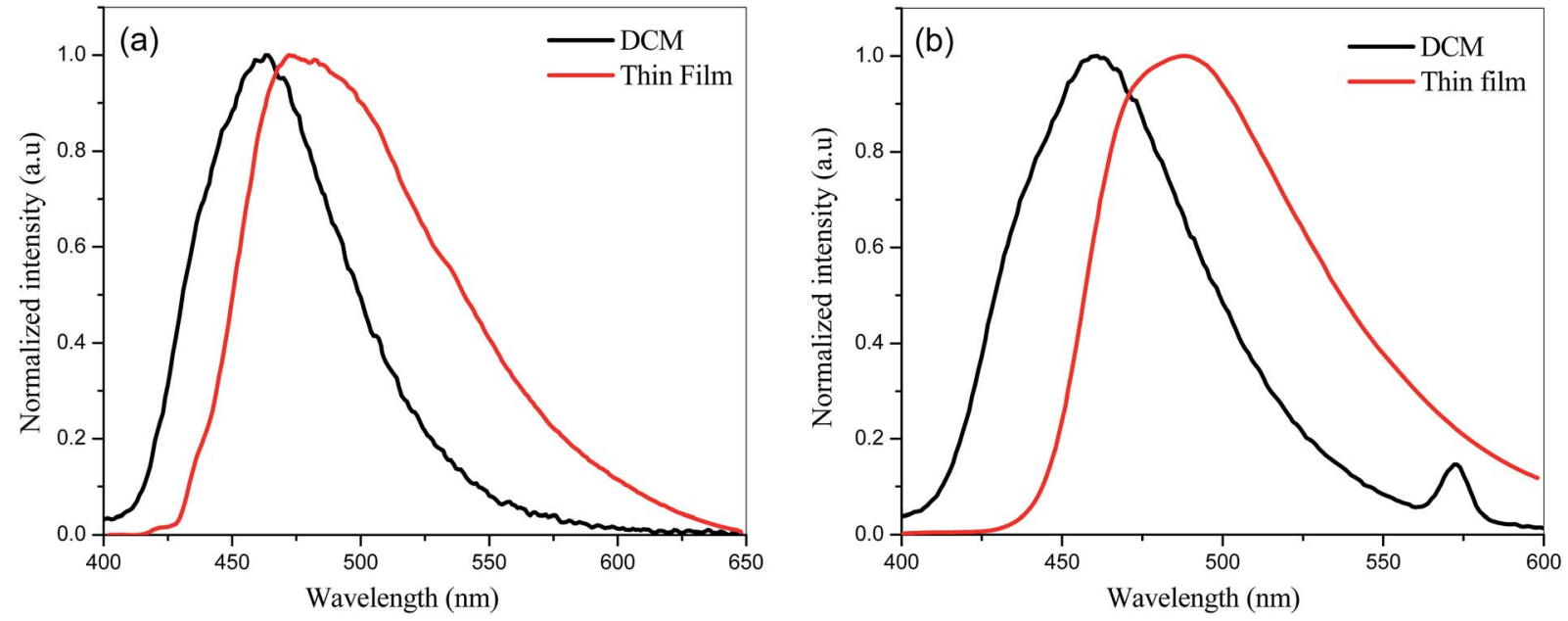

Fig. 3 Comparison of solution and thin film PL spectra for (a) compound 1 and (b) compound 2. 


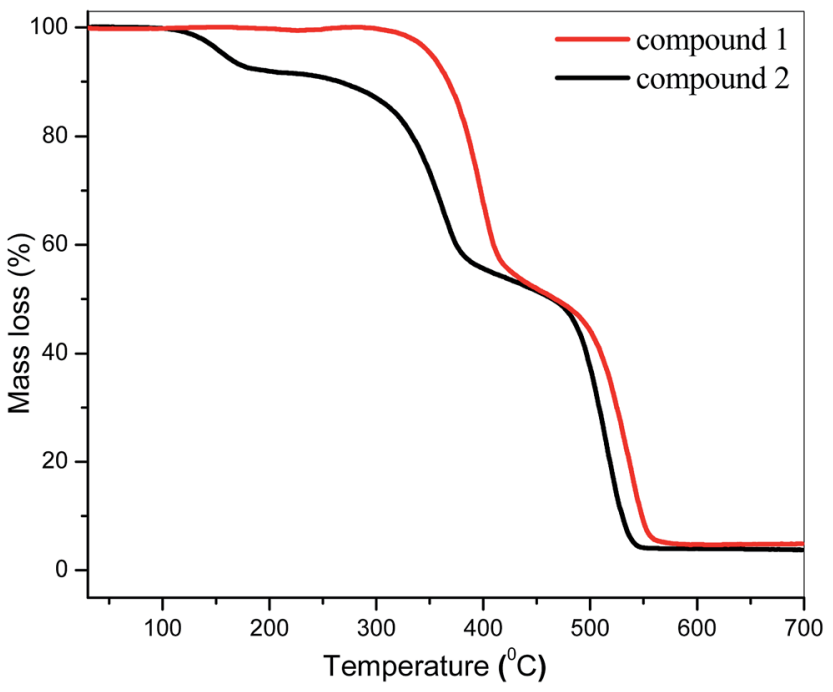

Fig. 4 TGA graphs showing mass loss (in percent) with temperature for 1 and 2.

$175{ }^{\circ} \mathrm{C}$ ) with $T_{\mathrm{d}}$ at $157{ }^{\circ} \mathrm{C}$. Thus it could be concluded that the known high thermal stabilities of the norbornane ring (m.p $85{ }^{\circ} \mathrm{C}$ ) and the adamantane ring (m.p. $270{ }^{\circ} \mathrm{C}$ ) imparted extra thermal stability to the synthesized new compounds $\mathbf{1} / \mathbf{2}$.

\subsection{Molecular packing}

To have more precise knowledge of the effect of rigid alkyl ring substituents on the emission behavior and thermal properties, it was essential to study the molecular interaction behavior of these newly developed coumarin derivatives. Thus efforts were devoted to grow single crystals for both derivatives, but success was achieved for compound $\mathbf{2}$ only and we could grow needle like single crystals from THF solution. Crystal refinement data for compound 2 can be found in the ESI (Table S1 $\dagger$ ). The crystal structure of compound 2 belongs to the monoclinic system having space group $P 2_{1} / c$. The crystal adopted a twist-like conformation of the $2 H$-benzo[h]chromen-2-one core and phenyl moieties with torsion angles of $52.01^{\circ}$ and $55.17^{\circ}$ (Fig. S4†). Previously we have reported that the parent coumarin shows a co-facial $\pi-\pi$ distance of $3.24 \AA$. In compound 2 , the adjacent layers in each column are placed in a cross fashion while adjacent columns showed head to head/tail to tail packing system (Fig. 5b and c). It is also interesting to note that substituting the hydroxyl group of the parent coumarin with the rigid and voluminous norbornane ring substituent helped the coumarin planar aromatic systems to achieve a cofacial $\pi-\pi$ distance of $4.01 \AA$ (Fig. 5b) between adjacent molecules which diminished the possibilities of $\pi-\pi$ interaction between adjacent layers. The absence of short/long wavelength emissions in thin film PL emission confirmed the absence of $\pi-\pi$ stacking. Although short range contacts such as $\mathrm{C}-\mathrm{H} \cdots \mathrm{O}(2.62 \AA)$, $\mathrm{C}-\mathrm{H} \cdots \mathrm{N}(2.73 \AA), \mathrm{C}-\mathrm{H} \cdots \mathrm{C}(2.89 \AA)$ were observed, these interactions did not contribute to ACQ. The carbonyl carbon which links the coumarin core with the norbornane ring did not show any interaction with the nearest heteroatom. These results and bathochromically shifted PL emission suggested the existence of small molecular interactions, but it could be concluded that rigid ring substitution was successful to reduce $\pi-\pi$ interactions and improved solid state PL emission compared to parent coumarin 3. Thus it could be assumed that the chances of nonradiative intermolecular interactions and long wavelength excimer emission were reduced through the current approach of structure engineering.
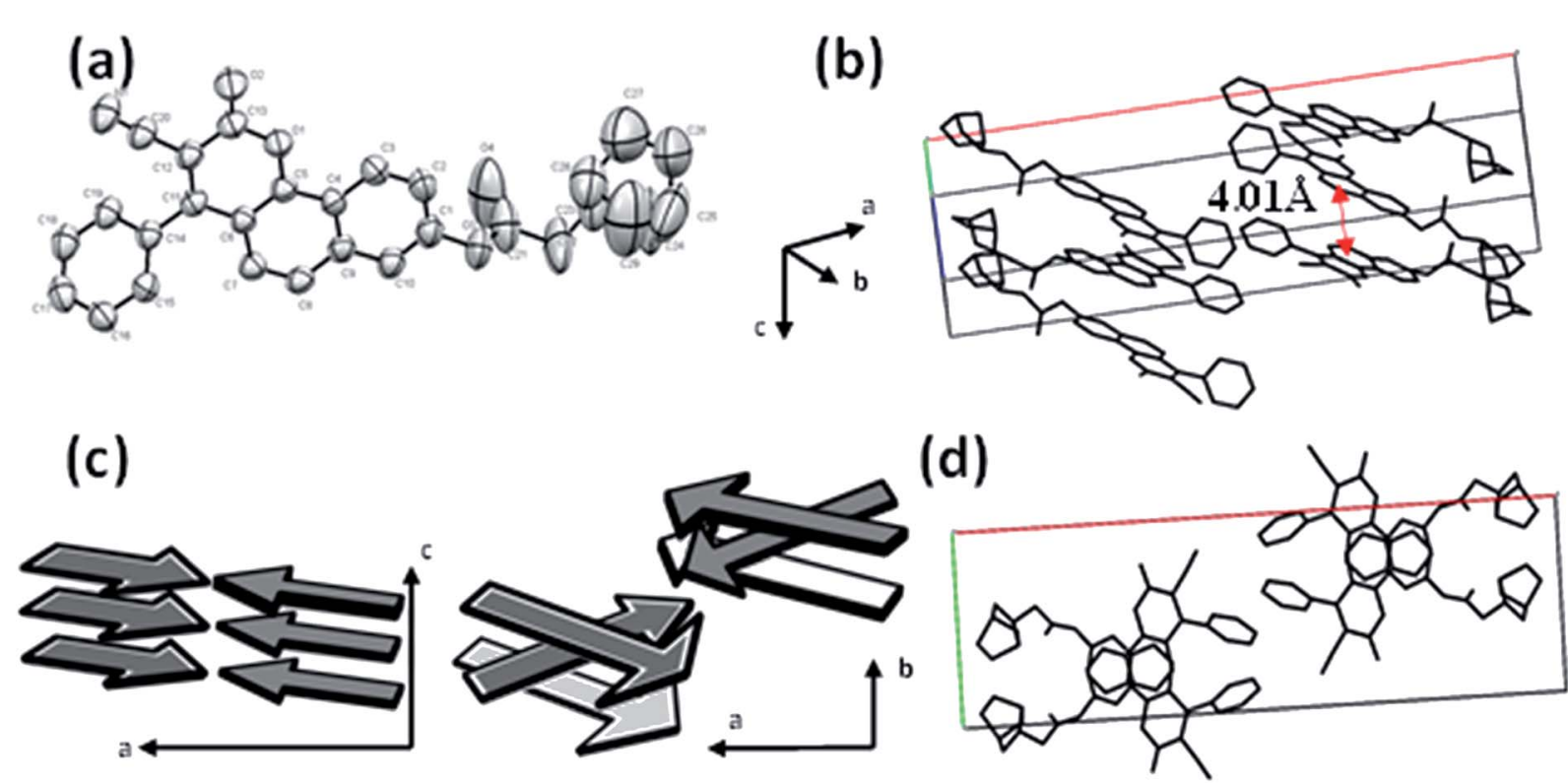

Fig. 5 (a) X-ray determined molecular structure of 2 using ORTEP diagram; (b) packing in the unit cell showing the inter-planar distance; (c) the arrows represent the stacking in the unit cell and indicate the direction of molecules from the aliphatic ring to the phenyl substituent and (d) crystal packing along the $c$ axis for compound 2 . 
Compound 1

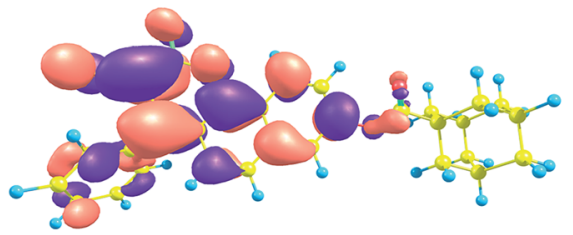

LUMO $=-2.77 \mathrm{eV}$

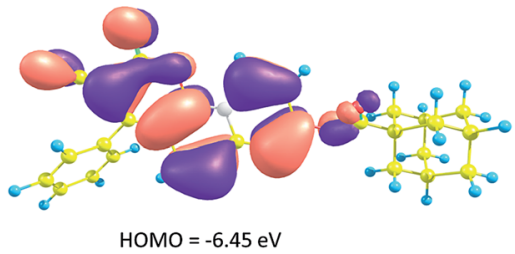

Compound 2

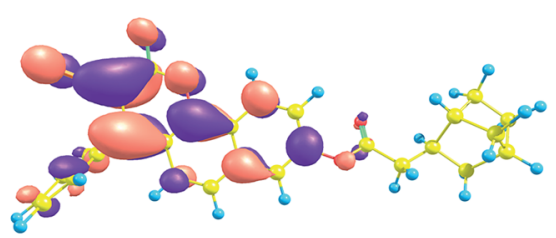

$L U M O=-2.80 \mathrm{eV}$

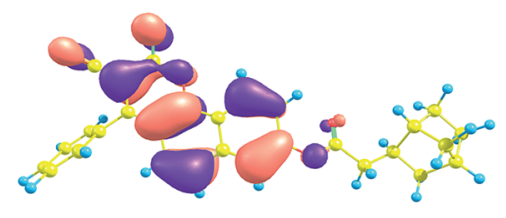

$\mathrm{HOMO}=-6.45 \mathrm{eV}$

Fig. 6 Theoretically calculated frontier molecular orbitals of compound 1 and 2 .

\subsection{Redox behavior and energy levels}

It is essential to acquire values of HOMO-LUMO energy levels of any compound to be utilized in light emitting devices along with the optical band gap. Cyclic voltammetry experiments were carried out in a dichloromethane solution of compound $\mathbf{1}$ and an acetonitrile solution of compound $2 . \mathrm{Ag} / \mathrm{AgCl}, \mathrm{Pt}$ disc and $\mathrm{Pt}$ wire were used as reference electrode, working electrode and counter electrode respectively. Both the compounds showed irreversible reduction peaks (Fig. S5 $†$ ) and oxidation peaks were not observed in the acquired potential window. From the calculation of reduction onset of $0.60 \mathrm{eV}$ for 1 and $0.59 \mathrm{eV}$ for 2, LUMO levels were assessed at $3.62 \mathrm{eV}$ and $3.65 \mathrm{eV}$ respectively. Optical band gaps of $2.83 \mathrm{eV}$ were calculated from the absorption onset of the DCM solution because of the absence of oxidation peaks in the acquired potential window of cyclic voltammetry provided HOMO energy levels at $-6.45 \mathrm{eV}$ and -6.48 $\mathrm{eV}$ for $\mathbf{1}$ and 2 respectively. It is interesting to note that the
HOMO-LUMO energy band gap of $2.83 \mathrm{eV}$ showed a shift of 0.05 $\mathrm{eV}$ from the previously reported values of $2.88 \mathrm{eV}$ for alkyl chain substituted coumarins. ${ }^{43}$ Thus the present approach is useful to eradicate greater shifts in optical band gap. These studies also show that one could expect improved emission properties of planar chromophores without noticeable modification in the precursor luminophore.

\subsection{DFT calculations}

Density functional theory calculations are effective to understand the spectral properties and for the analysis of molecular orbitals. Thus theoretical calculations using the B3LYP hybrid functional $^{59}$ and 6-311G (d,p) basis set on the Gaussian 09 (ref. 60) suite of programs have been carried out to analyze HOMOLUMO energy levels and distribution over the $2 H$-benzo[ $h]$ chromen-2-one core. Fig. 6 shows the corresponding HOMO and LUMO levels of 1 and 2 on optimized geometries (Fig. S6
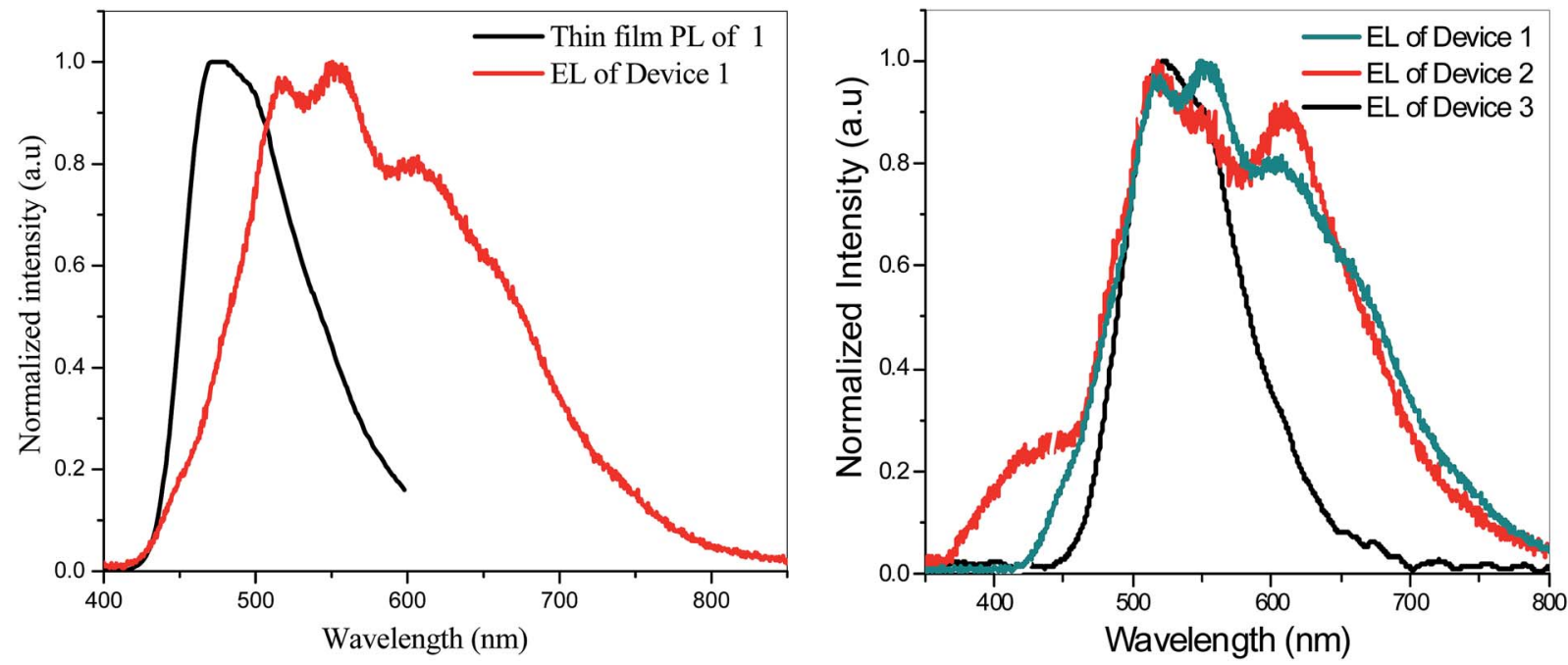

Fig. 7 (a) Comparison of thin film PL of 1 and EL of Device 1; and (b) comparison of EL spectra of Device 2 and Device 3 with Device 1. 


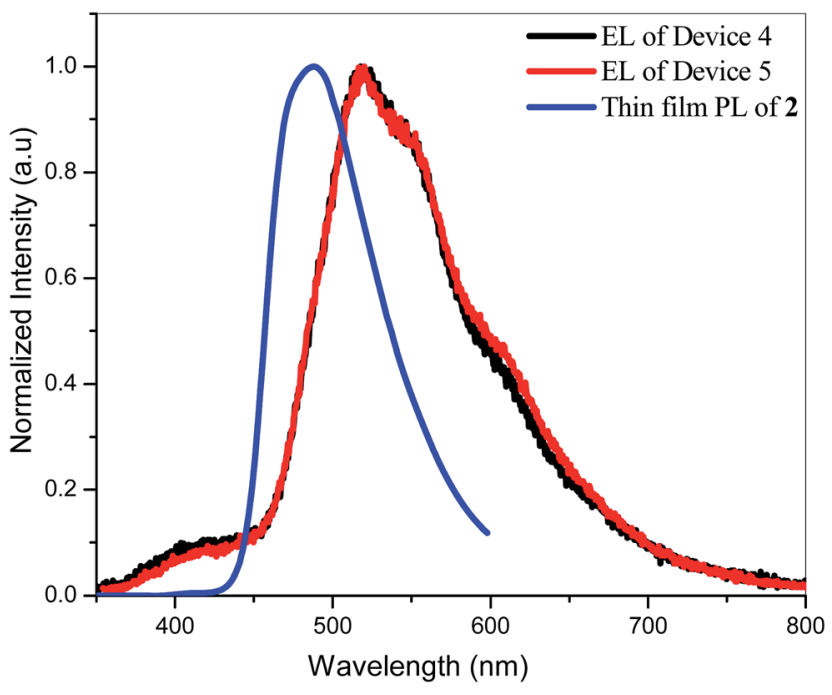

Fig. 8 EL comparison of Devices 4 and 5 with a thin film PL of compound 2 .

and $\mathrm{S} 7 \dagger$ ). These theoretical calculations helped to report that HOMO levels lie at $-6.45 \mathrm{eV}$ while LUMO energy levels lie at $2.77 \mathrm{eV}$ and $2.80 \mathrm{eV}$ for 1 and 2 respectively. Theoretically calculated HOMO levels are close to experimentally calculated levels, but LUMO energy levels showed a mismatch with theoretical calculations which could be expected because theoretical calculations were carried out for a gaseous single atom while experimental results were extracted from concentrated solutions of 1 and 2 . But it could be considered that theoretical band gaps of $3.67 \mathrm{eV}$ for 1 and $3.64 \mathrm{eV}$ for 2 are almost same. Similarly an equal optical band gap $(2.83 \mathrm{eV})$ for both compounds was observed from absorption onset in solution. UV-vis absorption properties for both optimized compounds have been calculated at the TD-B3LYP/6-311G (d,p) level of DFT. The UV-vis absorptions of both compounds were in good agreement with experimental absorption spectra (Fig. S8 and S9†). Thus an agreement between theoretical and experimental results could be understood.

\subsection{Thin film application}

To prove that it is possible to control $\pi-\pi$ stacking in planar emitting materials by introducing sterically bulky rigid ring substituents, we decided to fabricate OLED devices so that an agreement between the present approach and electroluminescence (EL) from devices could be settled. Firstly compound 1 was utilized as the sole emitting material in Device 1 having structure ITO/F4TCNQ (1 nm)/ $\alpha$-NPD (30 nm)/1 (35 nm)/BCP (6 $\mathrm{nm}) / \mathrm{Alq}_{3}(28 \mathrm{~nm}) / \mathrm{LiF}(1 \mathrm{~nm}) / \mathrm{Al}(150 \mathrm{~nm})$. The $\alpha$-NPD was used as the hole transport layer (HTL), BCP as the hole blocking layer (HBL) and $\mathrm{Alq}_{3}$ served as the electron transport layer (ETL). We reported earlier that a pristine layer of coumarin derivatives failed to show electroluminescence, rather an emission due to exciplex formed at the interface of the $\alpha$-NPD/coumarin derivative layer was observed at very high driving voltages. ${ }^{43}$ Thus to improve charge transport, 2,3,5,6-tetrafluoro-7,7,8,8- tetracyanoquinodimethane (F4TCNQ) was incorporated in Device 1 just before the $\alpha$-NPD layer. Device 1 showed a turn on voltage $\left(V_{\text {on }}\right)$ of $9 \mathrm{~V}$ having structured EL ranging between 450 nm-750 nm (Fig. 7a). It could be noted that the EL for compound 1 is red shifted from thin film PL emission (Fig. 7a), and peaks at 515 and $554 \mathrm{~nm}$ originated from 1. The long wavelength emission occurring at $610 \mathrm{~nm}$ that tails up to 750 $\mathrm{nm}$ is the exciplex emission ${ }^{\mathbf{4 3}}$ formed at the interface of $\alpha$-NPD/ 1. It could be noted that bathochromically shifted EL is a combination of PL of $\mathbf{1}$ and the exciplex emission forming at the $\alpha-\mathrm{NPD} / \mathbf{1}$ interface. Such red shifts in EL are observed commonly for OLED devices. Commission Internationale de L'Eclairage (CIE) approved reddish white light for the emission with color coordinates $(0.37,0.40)$.

Further, we advanced our studies to doped device structures for compounds 1 and 2 . Two more devices were fabricated with device structures as Device 2: ITO/ $\alpha$-NPD (30 nm)/s-CBP: 1 (35 $\mathrm{nm}) / \mathrm{BCP}(6 \mathrm{~nm}) / \mathrm{Alq}_{3}(28 \mathrm{~nm}) / \mathrm{LiF}(1 \mathrm{~nm}) / \mathrm{Al}(150 \mathrm{~nm})$ and Device 3: ITO/F4TCNQ $(1 \mathrm{~nm}) / \alpha-N P D(30 \mathrm{~nm}) / \mathrm{s}-\mathrm{CBP}: 1$ (35 nm)/BCP (6 $\mathrm{nm}) / \mathrm{Alq}_{3}(28 \mathrm{~nm}) / \mathrm{LiF}(1 \mathrm{~nm}) / \mathrm{Al}(150 \mathrm{~nm})$. Compound 1 was doped $1 \%$ by weight in s-CBP $\left(2,2^{\prime}, 7,7^{\prime}\right.$-tetrakis(carbazol-9-yl)9,9-spirobifluorene) host matrix and was utilized as emissive layer in both devices. Current density-voltage-luminescence (J$V-L$ ) characteristics (Fig. S10-S114 $\dagger$ ), current efficiencies and power efficiencies were calculated for all devices (Fig. S10S114 $\dagger$ ). Device 2 and Device 3 showed white light emission with $V_{\text {on }}$ of $10 \mathrm{~V}$ and $6 \mathrm{~V}$ respectively which proved that the F4TCNQ layer may have improved the charge transport and thus a lower turn on voltage was observed for Device 3. It is worth noting that the Device 2 architecture was not able to discard the formation of exciplex formation (Fig. 7b). Interestingly, EL from Device 3 was exactly a match for a thin film PL of compound 1 with a bathochromic shift of $37 \mathrm{~nm}$ (Fig. 7b). CIE coordinates for the emission were $(0.33,0.38)$. But exciplex emission was observed at voltages greater that $13 \mathrm{~V}$ which enabled us to report that exciplex formation is favorable at high working voltages (Fig. S15 $\dagger$ ). Similarly, we adapted doped device architecture for compound 2 and three more devices with 1\% doping were fabricated. Device 4: ITO/ $\alpha$-NPD $(30 \mathrm{~nm}) / \mathrm{s}-\mathrm{CBP}: 2(35 \mathrm{~nm}) / \mathrm{BCP}$ $(6 \mathrm{~nm}) / \mathrm{Alq}_{3}(28 \mathrm{~nm}) / \mathrm{LiF}(1 \mathrm{~nm}) / \mathrm{Al}(150 \mathrm{~nm})$ and Device 5 : ITO/F4TCNQ $(1 \mathrm{~nm}) / \alpha-N P D(30 \mathrm{~nm}) / \mathrm{s}-\mathrm{CBP}: 2$ (35 nm)/BCP (6 $\mathrm{nm}) / \mathrm{Alq}_{3}(28 \mathrm{~nm}) / \mathrm{LiF}(1 \mathrm{~nm}) / \mathrm{Al}(150 \mathrm{~nm})$. Devices 4 and 5 showed $V_{\text {on }}$ at $7 \mathrm{~V}$ and $6 \mathrm{~V}$. As expected, EL from all devices were red shifted compared to thin film PL at low driving voltages for Devices 4 and 5 (Fig. 8). It should be noted that peak emission was observed between $500 \mathrm{~nm}$ to $550 \mathrm{~nm}$ which further tailed up to $700 \mathrm{~nm}$ for Devices 4 and 5. Thus a white light with green tinge was observed. CIE coordinates for the emission were $(0.34$, 0.46) for Device 4. Peaks for exciplex emission were observed as a weak shoulder around $600 \mathrm{~nm}$ for Device 4 but exciplex emission was quite prominent at higher voltages for Device 5 (Fig. S16 $\dagger$ ). To confirm that $\mathrm{Alq}_{3}$ is not contributing to EL, Device $6[\mathrm{ITO} / \alpha$-NPD $(30 \mathrm{~nm}) / \mathrm{s}-\mathrm{CBP}: 1(35 \mathrm{~nm}) / \mathrm{PBD}(28 \mathrm{~nm}) / \mathrm{LiF}$ $(1 \mathrm{~nm}) / \mathrm{Al}(150 \mathrm{~nm})]$ containing PBD (2-(4-biphenyl)-5-(4-tertbutylphenyl)-1,3,4-oxadiazole) in place of $\mathrm{Alq}_{3}$ was fabricated. Device 6 showed a turn on voltage of 12-13 V and a broad EL centered on 515 was observed (Fig. S17†). Therefore, the long 
range emission in the electroluminescence was due to exciplex formation. Since the PBD is not a good electron transport layer for the present device, a high turn on voltage was observed with low electroluminescence.

\section{Conclusions}

In this study, a successful approach for controlling $\pi-\pi$ stacking and intermolecular interactions in planar coumarin derivatives was presented. Alicyclic norbornane and adamantyl rigid $3 \mathrm{D}$ arms were introduced to eradicate $\pi-\pi$ stacking and restore solid state emission. It was observed that emission wavelength and band gaps were unaffected with the introduction of these substitutions. But an investigation at the molecular level through single crystal structure analysis revealed a change in crystal packing and cofacial spacing. This provided molecules with AIEE property. Moreover thermal studies revealed that rigid cyclic rings impart better thermal stabilities than their linear chain counterpart. We were also able to prove using a combined theoretical and experimental approach that the present strategy of structure engineering through incorporation of peripheral rigid arms could enable planar molecules to tune their solid state optical properties, and those structurally modified materials can be used for OLED device applications with improved efficiency. As this manuscript reports some initial results to control aggregation and excimer formation, we believe that the present approach encourages the practice of using other bulky 3-D rigid ring substitutions as a helpful aspect to control the intermolecular interaction and excimer formation in planar luminophores. We also believe that a better device architecture would enable planar luminophores to be utilized as a pristine emissive layer for OLED application using the present approach. Presently we are working on device optimization for these coumarins and the outcome will be reported in the near future.

\section{Experimental section}

\subsection{General}

All the starting reactants were purchased from available commercial sources (Sigma-Aldrich, Merck and SD-Fine Chemicals) and were used as received. Anhydrous sodium sulfate $\left(\mathrm{Na}_{2} \mathrm{SO}_{4}\right)$, dichloromethane $\left(\mathrm{CH}_{2} \mathrm{Cl}_{2}\right)$ and other solvents were received from Merck and used as is. Spectroscopy grade solvents were utilized for photophysical studies. UV-vis and fluorescence spectra were recorded on a Shimadzu UV-2450 and a Cary Eclipse Fluorescence Spectrophotometer (Agilent Technologies) respectively. ${ }^{1} \mathrm{H}$ and ${ }^{13} \mathrm{C}$ NMR spectra were recorded on a Jeol ECX $500 \mathrm{MHz}$ NMR spectrometer. TMS was used as internal standard for ${ }^{1} \mathrm{H}$ NMR characterization. Mass spectroscopic studies were completed on a Bruker Maxis Impact HD instrument. FTIR studies were done on a Perkin-Elmer Spectrum 2 instrument. Solid state quantum yields were calculated on a Horiba Scientific Fluorolog-3. Thermal behavior studies were done using a NETZCH STA449 F1 instrument. The single crystal structure was solved using OLEX-2 software on Agilent single crystal XRD instrument. The electrochemical cyclic voltammetry (CV) was performed using a Metrohm Autolab electrochemical workstation in $0.1 \mathrm{M}$ tetrabutylammonium hexafluorophosphate $\left(\mathrm{Bu}_{4} \mathrm{NPF}_{6}\right)$ solution. The EL spectrum was measured with an Ocean Optics HR-2000CG UV-NIR. The current-voltage-luminescence $(I-V-L)$ characteristics have been measured with a luminance meter (LMT l-1009) interfaced with a Keithley 2400 programmable voltage-current digital source meter.

\subsection{Theoretical calculations}

Density functional theory calculations have been carried out to analyze the redox behavior of molecules. All calculations have been performed at the B3LYP/6-31G (d,p) level of density functional theory using the Gaussian 09 suite of programs.

\subsection{Device fabrication}

Indium-tin oxide (ITO) coated glass substrates with a sheet resistance of $20 \Omega \mathrm{sq}^{-1}$ were used as anodes which were patterned and cleaned by a conventional solution cleaning process. The substrates were further exposed to oxygen plasma to enhance the work function. All the layers were deposited by vacuum deposition at a base pressure of $1 \times 10^{-6}$ Torr with a deposition rate of $0.1 \mathrm{~A} \mathrm{~s}^{-1}$. The doped films were deposited by a co-evaporation process. The thicknesses of all the films were measured in situ by a quartz crystal thickness monitor. The size of each pixel was $2 \times 3 \mathrm{~mm}^{2}$. All further measurements were performed at room temperature and under an ambient atmosphere, without any encapsulation.

\section{Acknowledgements}

Financial support was received from the Department of Science and Technology, India (Grant No. SERB/F/2408/2012-13). Sunil Kumar is greatly thankful to University Grant Commission for providing doctoral scholarship. We are thankful to Dr T. J. Dhilip Kumar for his guidance to complete theoretical studies for present manuscript. We thankfully acknowledge the Director, IIT Mandi for research facilities. The support of Advanced Materials Research Center (AMRC), IIT Mandi, for sophisticated instrument facility is thankfully acknowledged. Punita Singh thanks Department of Science and Technology, India (Grant no. SR/WOS-A/CS-70/2012 from) for financial aid.

\section{References}

1 H. Li, Y. Guo, G. Li, H. Xiao, Y. Lei, X. Huang, J. Chen, H. Wu, J. Ding and Y. Cheng, J. Phys. Chem. C, 2015, 119, 6737-6748. 2 Z.-S. Wang, N. Koumura, Y. Cui, M. Takahashi, H. Sekiguchi, A. Mori, T. Kubo, A. Furube and K. Hara, Chem. Mater., 2008, 20, 3993-4003.

3 S. Kim, Q. Zheng, G. S. He, D. J. Bharali, H. E. Pudavar, A. Baev and P. N. Prasad, Adv. Funct. Mater., 2006, 16, 2317-2323.

4 S.-Y. Hsueh, C.-C. Lai, Y.-H. Liu, Y. Wang, S.-M. Peng and S.-H. Chiu, Org. Lett., 2007, 9, 4523-4526. 
5 C. W. Tang, S. A. van Slyke and C. H. Chen, J. Appl. Phys., 1989, 65, 3610-3616.

6 A. Poloek, C.-T. Chen and C.-T. Chen, J. Mater. Chem. C, 2014, 2, 1376-1380.

7 H. Sasabe and J. Kido, J. Mater. Chem. C, 2013, 1, 1699-1707. 8 B. O'Regan and M. Gratzel, Nature, 1991, 353, 737-740.

9 V. Roiati, R. Giannuzzi, G. Lerario, L. D. Marco, R. Agosta, R. Iacobellis, R. Grisorio, G. P. Suranna, A. Listorti and G. Gigli, J. Phys. Chem. C, 2015, 119, 6956-6965.

10 R. Singh, G. Pagona, V. G. Gregoriou, N. Tagmatarchis, D. Toliopoulos, Y. Han, Z. Fei, A. Katsouras, A. Avgeropoulos, T. D. Anthopoulos, M. Heeney, P. E. Keivanidis and C. L. Chochos, Polym. Chem., 2015, 6, 3098-3109.

11 P.-Y. Gu, Y. Ma, J.-H. He, G. Long, C. Wang, W. Chen, Y. Liu, Q.-F. Xu, J.-M. Lu and Q. Zhang, J. Mater. Chem. C, 2015, 3, 3167-3172.

12 C. R. Parker, E. Leary, R. Frisenda, Z. Wei, K. S. Jennum, E. Glibstrup, P. B. Abrahamsen, M. Santella, M. A. Christensen, E. A. Della Pia, T. Li, M. T. Gonzalez, X. Jiang, T. J. Morsing, G. Rubio-Bollinger, B. W. Laursen, K. Nørgaard, H. van der Zant, N. Agrait and M. B. Nielsen, J. Am. Chem. Soc., 2014, 136, 16497-16507.

13 S. Karmakar, S. Mardanya, S. Das and S. Baitalik, J. Phys. Chem. C, 2015, 119, 6793-6805.

14 Y. Li, P. Sonar, L. Murphy and W. Hong, Energy Environ. Sci., 2013, 6, 1684-1710.

15 Y. Tsutsui, T. Sakurai, S. Minami, K. Hirano, T. Satoh, W. Matsuda, K. Kato, M. Takata, M. Miura and S. Seki, Phys. Chem. Chem. Phys., 2015, 17, 9624-9628.

16 T. Mori, T. Nishimura, T. Yamamoto, I. Doi, E. Miyazaki, I. Osaka and K. Takimiya, J. Am. Chem. Soc., 2013, 135, 13900-13913.

17 N. Lin, X. Y. Liu, Y. Y. Diao, H. Xu, C. Chen, X. Ouyang, H. Yang and W. Ji, Adv. Funct. Mater., 2012, 22, 361-368.

18 G. Luchita, M. V. Bondar, S. Yao, I. A. Mikhailov, C. O. Yanez, O. V. Przhonska, A. E. Masunov and K. D. Belfield, ACS Appl. Mater. Interfaces, 2011, 3, 3559-3567.

19 T. M. Figueira-Duarte and K. Müllen, Chem. Rev., 2011, 111, 7260-7314.

20 Q. Zou, Y. Zhao, N. S. Makarov, J. Campo, H. Yuan, D.-C. Fang, J. W. Perry and F. Wu, Phys. Chem. Chem. Phys., 2012, 14, 11743-11752.

21 J.-S. Yang and J.-L. Yan, Chem. Commun., 2008, 1501-1512.

22 Y.-J. Choi, M. Park, D.-Y. Kim, C.-H. Hsu, S.-H. Hwang and K.-U. Jeong, J. Phys. Chem. Lett., 2015, 6, 887-892.

23 H. Y. Woo, B. Liu, B. Kohler, D. Korystov, A. Mikhailovsky and G. C. Bazan, J. Am. Chem. Soc., 2005, 127, 14721-14729.

24 J. Wang, Y. Zhao, C. Dou, H. Sun, P. Xu, K. Ye, J. Zhang, S. Jiang, F. Li and Y. Wang, J. Phys. Chem. B, 2007, 111, 5082-5089.

25 B. S. Gaylord, S. Wang, A. J. Heeger and G. C. Bazan, J. Am. Chem. Soc., 2001, 123, 6417-6418.

26 C. L. Chiang, S. M. Tseng, C. T. Chen, C. P. Hsu and C. F. Shu, Adv. Funct. Mater., 2008, 18, 248-257.
27 C.-T. Chen, Y. Wei, J.-S. Lin, M. V. R. K. Moturu, W.-S. Chao, Y.-T. Tao and C.-H. Chien, J. Am. Chem. Soc., 2006, 128, 10992-10993.

28 S. Thiery, D. Tondelier, C. Declairieux, B. Geffroy, O. Jeannin, R. Métivier, J. Rault-Berthelot and C. Poriel, J. Phys. Chem. C, 2015, 119, 5790-5805.

29 W. Z. Yuan, P. Lu, S. Chen, J. W. Y. Lam, Z. Wang, Y. Liu, H. S. Kwok, Y. Ma and B. Z. Tang, Adv. Mater., 2010, 22, 2159-2163.

30 J. N. Moorthy, P. Natarajan, P. Venkatakrishnan, D.-F. Huang and T. J. Chow, Org. Lett., 2007, 9, 5215-5218.

31 S. Hecht and J. M. J. Fréchet, Angew. Chem., Int. Ed., 2001, 40, 74-91.

32 Z. Xie, B. Yang, F. Li, G. Cheng, L. Liu, G. Yang, H. Xu, L. Ye, M. Hanif, S. Liu, D. Ma and Y. Ma, J. Am. Chem. Soc., 2005, 127, 14152-14153.

33 J. Luo, Z. Xie, J. W. Y. Lam, L. Cheng, H. Chen, C. Qiu, H. S. Kwok, X. Zhan, Y. Liu, D. Zhu and B. Z. Tang, Chem. Commun., 2001, 1740-1741.

34 Y. Hong, J. W. Y. Lam and B. Z. Tang, Chem. Commun., 2009, 4332-4353.

35 B.-K. An, S.-K. Kwon, S.-D. Jung and S. Y. Park, J. Am. Chem. Soc., 2002, 124, 14410-14415.

36 M. Cai, Z. Gao, X. Zhou, X. Wang, S. Chen, Y. Zhao, Y. Qian, N. Shi, B. Mi, L. Xie and W. Huang, Phys. Chem. Chem. Phys., 2012, 14, 5289-5296.

37 X. Zhang, Z. Chi, H. Li, B. Xu, X. Li, W. Zhou, S. Liu, Y. Zhang and J. Xu, Chem.-Asian J., 2011, 6, 808-811.

38 G. Iasilli, A. Battisti, F. Tantussi, F. Fuso, M. Allegrini, G. Ruggeri and A. Pucci, Macromol. Chem. Phys., 2014, 215, 499-506.

39 X. Li, X. Zhang, Z. Chi, X. Chao, X. Zhou, Y. Zhang, S. Liu and J. Xu, Anal. Methods, 2012, 4, 3338-3343.

40 B. Xu, Z. Chi, H. Li, X. Zhang, X. Li, S. Liu, Y. Zhang and J. Xu, J. Phys. Chem. C, 2011, 115, 17574-17581.

41 J. Huang, N. Sun, P. Chen, R. Tang, Q. Li, D. Ma and Z. Li, Chem. Commun., 2014, 50, 2136-2138.

42 K.-Y. Shih, T.-S. Hsiao, S.-L. Deng and J.-L. Hong, Macromolecules, 2014, 47, 4037-4047.

43 S. Kumar, P. Singh, R. Srivastava, R. R. Koner, A. Pramanik, J. Mathew, S. Sinha, M. Rawat, R. S. Anand and S. Ghosh, J. Mater. Chem. C, 2014, 2, 6637-6647.

44 Q. Zeng, D. Wu, H. Ma, C. Shu, Y. Li and C. Wang, CrystEngComm, 2006, 8, 189-201.

45 N. Pannier and W. Maison, Eur. J. Org. Chem., 2008, 2008, 1278-1284.

46 E. Galoppini and R. Gilardi, Chem. Commun., 1999, 173-174. 47 W. Maison, J. V. Frangioni and N. Pannier, Org. Lett., 2004, 6, 4567-4569.

48 S. J. Cowling, A. W. Hall and J. W. Goodby, Liq. Cryst., 2005, 32, 1483-1498.

49 M. Tominaga, K. Katagiri and I. Azumaya, Cryst. Growth Des., 2009, 9, 3692-3696.

50 S. R. Amrutha and M. Jayakannan, Macromolecules, 2007, 40, 2380-2391.

51 M. Tasior, D. Kim, S. Singha, M. Krzeszewski, K. H. Ahn and D. T. Gryko, J. Mater. Chem. C, 2015, 3, 1421-1446. 
52 X. Liu, J. M. Cole and K. S. Low, J. Phys. Chem. C, 2013, 117, 14723-14730.

53 P. Kotchapadist, N. Prachumrak, T. Sunonnam, S. Namuangruk, T. Sudyoadsuk, T. Keawin, S. Jungsuttiwong and V. Promarak, Eur. J. Org. Chem., 2015, 2015, 496-505.

54 M.-T. Lee, C.-K. Yen, W.-P. Yang, H.-H. Chen, C.-H. Liao, C.-H. Tsai and C. H. Chen, Org. Lett., 2004, 6, 1241-1244.

55 J. N. Moorthy, P. Venkatakrishnan, P. Natarajan, D.-F. Huang and T. J. Chow, J. Am. Chem. Soc., 2008, 130, 17320-17333.
56 X. Chen, X. Zhang and G. Zhang, Chem. Commun., 2015, 51, 161-163.

57 C. Reichardt, Chem. Rev., 1994, 94, 2319-2358.

58 M. Kimura, S. Kuwano, Y. Sawaki, H. Fujikawa, K. Noda, Y. Taga and K. Takagi, J. Mater. Chem., 2005, 15, 2393-2398. 59 A. D. Becke, J. Chem. Phys., 1993, 98, 5648-5652.

60 M. Frisch, G. Trucks, H. B. Schlegel, G. Scuseria, M. Robb, J. Cheeseman, G. Scalmani, V. Barone, B. Mennucci and G. Petersson, Gaussian 09, Revision A. 02, Gaussian, Inc., Wallingford, CT, 2009. 